$\mathrm{mL})$ at diagnosis $(2,574)$ than those reporting internet $(6,275)$ or place $(11,745)$ only venues.

Conclusions MSM meeting sex partners at both venue types may be at greater risk for HIV transmission based on number of sex partners, sex under influence of alcohol, and chlamydia infection. Lower viral load may suggest later diagnosis which increases transmission risk. Exploring this population may improve control strategies.

\section{P04.09 ASSOCIATION OF GENDER OF SEXUAL PARTNERS WITH WOMEN'S SEXUALLY TRANSMITTED INFECTION RISK}

HS Harbison*, EL Austin, EW Hook, CM Muzny. Division of Infectious Diseases, University of Alabama at Birmingham

10.1136/sextrans-2015-052270.263

Background Previous data suggest that women who have sex with women and men (WSWM) have the highest STI risk compared to other female sexual behaviour groups. We compared risk behaviours and STI rates among women who have sex with women (WSW), WSWM, women with a single male partner (WSM), and women with multiple male partners. We hypothesised a continuum of STI risk with WSW having the lowest risk, followed by WSM with one partner, WSM with multiple partners, and WSWM.

Methods A secondary analysis of data from two studies of African American women evaluated at a Birmingham, Alabama STD clinic was performed. One study included exclusive WSW ( $\mathrm{n}=$ $78)$ and WSWM $(n=85)$ during the preceding year while the other evaluated WSM ( $\mathrm{n}=91$ with one male partner and $\mathrm{n}=$ 78 with multiple male partners) during this timeframe. All participants completed a questionnaire and were tested for STIs.

Results Groups did not differ by education, employment status, or recent alcohol/drug use. WSWM and WSM with multiple partners were more likely to report prior incarceration, transactional sex, and new/casual partner (s) within the past month. Rates of chlamydia and gonorrhoea were significantly different across groups with WSM more likely to have chlamydia and WSWM and WSM more likely to have gonorrhoea. In contrast, WSW and WSWM were more likely to have trichomoniasis than WSM. With the exception of WSW (25\%), HSV-2 seroprevalence was high $(>50 \%)$ among all groups. WSWM and WSM with multiple partners were more likely to report condom use at their last sexual encounter than WSM with single male partners and WSW.

Conclusion Among women attending an STD clinic, STIs were common in all groups however prevalence appears to vary in association with partner gender and number. Even in STD clinics, partner gender and number are important factors to consider in sexual health counselling.

Disclosure of interest statement Funding for original research was received from a Development Award from the American Sexually Transmitted Disease Association. No pharmaceutical grants were received in the development of this study.

\section{P04.10 THE LINK BETWEEN POPULATION SEXUAL BEHAVIOUR AND HIV PREVALENCE IN SUB-SAHARAN AFRICA}

1,2,3R Omori ${ }^{*},{ }^{2,3,4} \mathrm{~L}$ Abu-Raddad. ${ }^{1}$ Hokkaido University; ${ }^{2}$ Weill Cornell Medical College Qatar; ${ }^{3}$ Weill Cornell Medical College; ${ }^{4}$ Fred Hutchinson Cancer Research Center

$10.1136 /$ sextrans-2015-052270.264
Introduction The patterns of sexual partnering and structure of sexual networks should shape HIV transmission in human populations. We examined the empirical association between population casual-sex behaviour and HIV prevalence, and the empirical associations between different measures of casual-sex behaviour. Methods An ecologic study design was applied to the nationallyrepresentative data of the Demographic and Health Surveys in 25 countries in sub-Saharan Africa. Spearman rank correlation was used to assess the association between HIV prevalence and means and variances of the number of casual-sex partners. Spearman rank correlation was also used to assess the associations between the different means, different variances, and means and variances of the number of casual-sex partners.

Results Correlations between HIV prevalence and means and variances of the number of casual-sex partners were positive, but small and statistically insignificant. The majority of correlations across means and variances of the number of casual-sex partners were positive, large, and statistically significant. However, all correlations between the means, as well as variances, and the variance of unmarried females were weak and statistically insignificant. Population casual-sex behaviour was not predictive of HIV prevalence across these African countries. Nevertheless, the strong correlations across means and variances suggest that selfreported sexual data are self-consistent and may convey credible information.

Conclusion Self-reported population sexual behaviour was not found predictive of HIV prevalence, but appears inherently selfconsistent and with valid information content. Unmarried female behaviour seems puzzling, but could be playing an influential role in HIV transmission patterns.

Disclosure of interest statement No pharmaceutical grants were received in the development of this study.

\section{P04.11 ESTIMATION OF NON-COHABITING SEX PARTNERING IN SUB-SAHARAN AFRICA}

${ }^{1,2,3}{ }^{R}$ Omori ${ }^{*}{ }^{2} \mathrm{H}$ Chemaitelly, ${ }^{2,3,4} \mathrm{~L}$ Abu-Raddad. ${ }^{1}$ Hokkaido University; ${ }^{2}$ Weill Cornell Medical College - Qatar; ${ }^{3}$ Weill Cornell Medical College; ${ }^{4}$ Fred Hutchinson Cancer Research Center

\subsection{6/sextrans-2015-052270.265}

Introduction Understanding the patterns of sexual partnering and structure of sexual networks is essential for understanding the epidemiological dynamics of sexually transmitted infections (STI) in human populations. This study aimed to develop an analytical understanding of non-cohabiting sex partnering in subSaharan Africa (SSA) by utilising nationally-representative sexual behaviour data.

Methods A non-homogenous Poisson stochastic process model was used to describe the dynamics of non-cohabiting sex. The model was applied to 25 countries in SSA and was fitted to Demographic and Health Survey (DHS) data. The country-specific means and variances of the distributions of number of noncohabiting partners were estimated.

Results The model showed robust fits to the empirical distributions stratified by country, marital status and sex. The median across all country-specific means was highest for unmarried males at 0.574 non-cohabiting partners over the last 12 months, followed by that of unmarried females at 0.337 , married males at 0.192 , and married females at 0.038 . The median of variances was highest for unmarried males at 0.127 , followed by married males at 0.057 , unmarried females at 0.003 , and married females at 0.000 . The largest variability in means across countries was 
for unmarried males (0.103 to 1.206$)$, and the largest variability in variances was among unmarried females (0.000 to 1.994).

Conclusion Robust fits of our model to the empirical sexual behaviour data suggest that non-cohabiting sex partnering appears to be a random "opportunistic" phenomenon. Unmarried individuals have larger means than their married counterparts, and males have larger means than females. Unmarried individuals appear to play a key role in driving heterogeneity in sexual networks and STIs epidemiology.

Disclosure of interest statement No pharmaceutical grants were received in the development of this study.

\section{P04.12 FRONT-TO-BACK WIPING AND DABBING BEHAVIOUR WIPING POST-TOILET SIGNIFICANTLY ASSOCIATED WITH ANAL NEOPLASIA AND HR-HPV CARRIAGE IN A COHORT OF WOMEN WITH A HISTORY OF AN HPV- MEDIATED GYNAECOLOGICAL NEOPLASIA}

${ }^{1}$ Steve Simpson*, 2,3Penny Blomfield, ${ }^{4}$ Amanda Dennis, ${ }^{5}$ Sepehr Tabrizi, ${ }^{1,3}$ Richard Turner. ${ }^{1}$ Menzies Institute for Medical Research, University of Tasmania, Hobart Australia; ${ }^{2}$ Gynaecologic Oncology, Royal Hobart Hospital, Hobart, Australia; ${ }^{3}$ School of Medicine, University of Tasmania, Hobart, Australia; ${ }^{4}$ Gynaecologic Oncology, Launceston General Hospital, Launceston, Australia; ${ }^{5}$ Department of Microbiology and Infectious Diseases, The Royal Women's Hospital, Parkville, Australia; ${ }^{6}$ School of Medicine, University of Tasmania, Hobart, Australia

\subsection{6/sextrans-2015-052270.266}

Background Anal cancer is an HPV-mediated neoplasia of the squamous epithelium of the anus. A hitherto unexplained feature of anal cancer epidemiology is its much higher frequency among women, who comprise nearly two thirds of cases.

Methods Cross-sectional study of women with a history of an HPV-mediated gynaecological neoplasia in Tasmania, Australia. Women presenting for follow-up gynaecological care had anal swab samples taken for anal cytology by Hologic Liquid ThinPrep and HR-HPV typing. Women with abnormal anal cytology were invited for high-resolution anoscopy (HRA). Covariates of anal outcomes evaluated by log-binomial and log-multinomial regression.

Results 163 women had anal swabs taken and comprised the study sample, of whom $40.0 \%$ had abnormal cytology $(28.2 \%$ high-grade). Of the 50 women with abnormal anal cytology having an HRA, $64.0 \%$ had abnormal histology (26.0\% high-grade). Of the 120 women having anal HPV typing done, $31.7 \%$ had HR-HPV carriage, the most common types being 51 (11.5\%) and $16(9.2 \%)$.

In addition to some known anal cancer risk factors, we found front-to-back (FTB) wiping was associated with significantly increased prevalence of cytological and histological abnormality and HR-HPV carriage, while dabbing post-toilet was significantly decreased prevalences of all anal outcomes, all robust to adjustment.

Conclusions We have shown here that post-toilet wiping behaviours, specifically FTB and dabbing, significantly modulated the prevalence of anal cytological, histological and HR-HPV carriage outcomes. While the cross-sectional design precludes ascription of causality, the biological plausibility of such an autoinoculation pathway might help explain the preponderance of anal cancer among women.

Conflicts of interest We have no conflicts to disclose.

\section{P04.13 THE SEXUAL HEALTH LITERACY OF THE STUDENT POPULATION OF THE UNIVERSITY OF TASMANIA: RESULTS OF THE RUSSL STUDY}

${ }^{1}$ Steve Simpson* ${ }^{2}$ Christine Clifford, ${ }^{3}$ Kaz Ross, ${ }^{4}$ Neil Sefton, ${ }^{5}$ Louise Owen, ${ }^{1}$ Leigh Blizzard, ${ }^{1,4}$ Richard Turner. 'Menzies Institute for Medical Research, University of Tasmania, Hobart Australia; ${ }^{2}$ School of Psychology, University of Tasmania, Hobart Australia; ${ }^{3}$ School of Humanities, University of Tasmania, Hobart Australia; ${ }^{4}$ School of Medicine, University of Tasmania, Hobart Australia; ${ }^{5}$ Sexual Health Service Tasmania, Tasmanian Department of Health and Human Services, Hobart Australia

\section{$10.1136 /$ sextrans-2015-052270.267}

Introduction/background/issues Evidence suggests a varied level of sexual health literacy (SHL) among university student populations. Accordingly, we evaluated the SHL among students at the University of Tasmania.

Methods Students were invited to complete an anonymous online questionnaire.

Recruitment was during August/September 2013. SHL was assessed by ARCSHS National Survey of Australian Secondary Students and Sexual Health and the Sexual Health Questionnaire. Predictors of literacy scores were evaluated by linear regression.

Results/discussions The study recruited 1,786 participants, or $8.2 \%$ of the student population, of similar composition to the general university population.

Female sex, older age, and sexual education and increased communication on sexual topics were significant predictors of literacy scores. Literacy increased with sexual experience (ever sex, earlier age of sexual debut, partner number, diversity of sexual activity). Students in medical/nursing disciplines had the highest SHL.

Compared to Australian/New Zealander students, overseasborn students had significantly lower ARC $(-3.6 \%, \mathrm{p}<0.001)$ and SHS $(-4.2 \%, \mathrm{p}<0.001)$, this driven by Malaysian, Indian and Chinese students. Compared to agnostic/atheist-identifying students, those of Buddhist (ARC: $-5.4 \%, p=0.014$; SHS: $6.7 \%, \mathrm{p}=0.002$ ), Muslim (ARC: $-16.5 \%, \mathrm{p}<0.001$; SHS: $13.4 \%, \mathrm{p}=0.001$ ) and Protestant (ARC: $-2.3 \%, \mathrm{p}=0.023$; SHS: $-4.4 \%, \mathrm{p}<0.001)$ identifications had markedly lower SHL. Importantly, many of these associations persisted on adjustment for age, sex, sexual education and sexual experience.

Conclusions/implications This study, one of the first targeting among university students in Australia, found a varied SHL by sex, age, sexual education and sexual experience, as well as by birthplace and religious affiliation. These findings have applications in orientation and education programs at Australian universities.

Key message This study substantiates the ongoing deficits in sexual health literacy in some minority religious/ethnic/birthplace populations, suggesting some role for remedial sexual health education in university orientation programs may be appropriate.

\section{P04.14 PREVALENCE AND CONSISTENCY OF OPINIONS ON SAME-SEX PARTNERSHIPS OVER 12 YEARS IN A NEW ZEALAND BIRTH COHORT}

${ }^{1} \mathrm{~J}$ Connor, ${ }^{2} \mathrm{E}$ Burgess, ${ }^{2} \mathrm{~J}$ Cresswell, ${ }^{1} \mathrm{~A}$ Righarts, ${ }^{1} \mathrm{~N}$ Dickson. ${ }^{2}$ Department of Preventive and Social Medicine, University of Otago, Dunedin, New Zealand; '² London School of Hygiene and Tropical Medicine, London, UK

10.1136/sextrans-2015-052270.268 